\title{
Multiobjective Optimization of Turning Cutting Parameters for J-Steel Material
}

\author{
Adel T. Abbas, ${ }^{1}$ Karim Hamza, ${ }^{2}$ Mohamed F. Aly, ${ }^{3}$ and Essam A. Al-Bahkali ${ }^{1}$ \\ ${ }^{1}$ Department of Mechanical Engineering, King Saud University, P.O. Box 800, Riyadh 11421, Saudi Arabia \\ ${ }^{2}$ Department of Mechanical Engineering, University of Michigan, Ann Arbor, MI 48105, USA \\ ${ }^{3}$ Department of Mechanical Design, American University in Cairo, New Cairo, Cairo 11835, Egypt
}

Correspondence should be addressed to Adel T. Abbas; atabbas1954@yahoo.com

Received 11 January 2016; Revised 20 March 2016; Accepted 23 March 2016

Academic Editor: Wenbin Yi

Copyright (C) 2016 Adel T. Abbas et al. This is an open access article distributed under the Creative Commons Attribution License, which permits unrestricted use, distribution, and reproduction in any medium, provided the original work is properly cited.

\begin{abstract}
This paper presents a multiobjective optimization study of cutting parameters in turning operation for a heat-treated alloy steel material (J-Steel) with Vickers hardness in the range of HV 365-395 using uncoated, unlubricated Tungsten-Carbide tools. The primary aim is to identify proper settings of the cutting parameters (cutting speed, feed rate, and depth of cut) that lead to reasonable compromises between good surface quality and high material removal rate. Thorough exploration of the range of cutting parameters was conducted via a five-level full-factorial experimental matrix of samples and the Pareto trade-off frontier is identified. The tradeoff among the objectives was observed to have a "knee" shape, in which certain settings for the cutting parameters can achieve both good surface quality and high material removal rate within certain limits. However, improving one of the objectives beyond these limits can only happen at the expense of a large compromise in the other objective. An alternative approach for identifying the trade-off frontier was also tested via multiobjective implementation of the Efficient Global Optimization (m-EGO) algorithm. The m-EGO algorithm was successful in identifying two points within the good range of the trade-off frontier with $36 \%$ fewer experimental samples.
\end{abstract}

\section{Introduction}

Heat-treated alloy steels exhibit many attractive properties, such as wear resistance, high strength, and high thermal stability. Examples of uses of heat-treated alloy steels include dies, automotive structures, bearings, and gears. In a highly competitive world, there is much need for optimizing the manufacturing and processing technologies to achieve highquality products at low cost and high productivity. Focus of this research is on optimizing the cutting parameters of CNCturning operations of J-Steel material in order to achieve (i) high-quality surface finish (which translates to product quality) and (ii) high material removal rate (which translates to productivity). Cost is not modeled in this study due to it being (at least partially) correlated with productivity and due to several uncertainties that can be site-specific (e.g., cost of labor, energy, and transport).

Examples of notable related work in the literature of machinability of other types of hardened steels include [1-3], and a review of state of the art and identification of active research areas is summarized in [4]. The primary control parameters (also termed "design variables" within an optimization framework) in CNC turning are the machining parameters (cutting speed, feed rate, and depth of cut), material, and geometry of the cutting tools and cutting fluids. Hard-turning operations require no cutting fluids, which is advantageous in terms of cost (purchase and disposal of cutting fluids), environmental impact (elimination of the need to posttreat the cutting fluids for safe disposal), and process tenability (fewer parameters to adjust). By selecting a set of standard Tungsten-Carbide insert tools, thereby considering the tooling as a fixed parameter, this study conducts a thorough examination of the three machining parameters and how they relate to the trade-off between surface quality and material removal rate.

Tuning of the cutting parameters is conducted in this paper within the context of Pareto-optimality in order to find good compromises between surface quality and process 

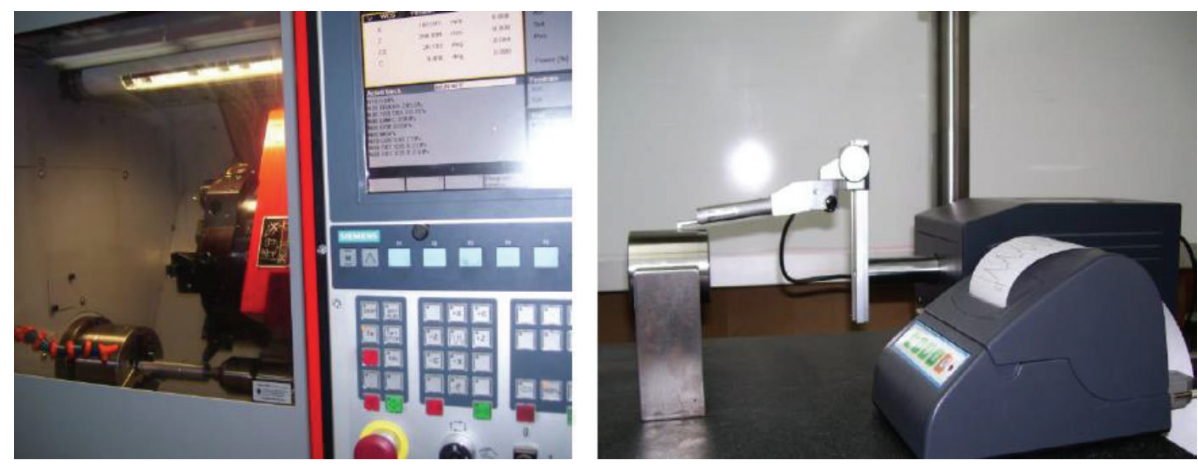

FIGURE 1: Test rig for turning and surface roughness measurement.

productivity. The tuning is done via "dense sampling" (fivelevel full-factorial design of experiments) as well as multiobjective implementation of an optimization technique from metamodeling based design optimization, "Efficient Global Optimization" (EGO) [5-8]. EGO seeks to locate the optimum of a processes while conducting as few experiments as possible. This is done via incrementally updating a Gaussian process metamodel that approximates the behavior of the actual process. Multiobjective versions of EGO such as ParEGO [9] and m-EGO, which is some of the authors' earlier work in $[10,11]$, have been proposed and tested via numerical models. To the best of the authors' knowledge, the present study is the first experimentation with utilizing m-EGO in real machining experiments.

This paper started with a brief introduction and motivation of the performed research. The rest of the paper is organized as follows. Section 2 provides details of the experimental setup and material to be machined. Section 3 showcases the results from a five-level full-factorial experimental matrix (125 samples). Section 4 presents the mEGO approach for identification of the Pareto trade-off frontier. Section 5 discusses the results and implications from both approaches, and then the paper concludes with a brief summary.

\section{Experimental Setup}

EMCO Concept Turn 45 CNC lathe equipped with Sinumeric 840-D was used to conduct experimental work. An uncoated Tungsten-Carbide insert was clamped with the tool holder to carry out this work. The specifications for insert and tool holder are TR-V13JBL 2020K and TR-VB1304-F. Clearance angle, cutting edge angle, and nose radius are maintained by $7^{\circ}, 75^{\circ}$, and $0.4 \mathrm{~mm}$, respectively. After machining, a surface roughness tester TESA was used to measure the surface roughness over a $50 \mathrm{~mm}$ length of the machined surface. Photos of the test rig are shown in Figure 1.

Chemical composition of J-Steel material is listed in Table 1. The heat treatment for involved Austenitizing at $850^{\circ} \mathrm{C}$ for $4-5$ hours, quenching in oil, tempered at $600-630^{\circ} \mathrm{C}$ for 6-7 hours and then air-cooled. Hardness was HV 365-395.
TABLE 1: Chemical composition of the J-Steel material.

\begin{tabular}{lccccccccc}
\hline Element & $\mathrm{C}$ & $\mathrm{Si}$ & $\mathrm{Mn}$ & $\mathrm{Ni}$ & $\mathrm{Cr}$ & $\mathrm{Mo}$ & $\mathrm{V}$ & $\mathrm{S}$ & $\mathrm{P}$ \\
\hline Minimum\% & 0.30 & 0.10 & 0.20 & 2.7 & 0.7 & 0.40 & $<0.25$ & $\leq 0.015$ & $\leq 0.015$ \\
Maximum\% & 0.4 & 0.35 & 0.7 & 3.3 & 1.2 & 0.7 & & & \\
\hline
\end{tabular}

TABLE 2: Design variables values in five-level full-factorial matrix.

\begin{tabular}{lccc}
\hline Level & $\begin{array}{c}\text { Cutting speed } \\
x_{1}(\mathrm{~m} / \mathrm{min})\end{array}$ & $\begin{array}{c}\text { Depth of cut } \\
x_{2}(\mathrm{~mm})\end{array}$ & $\begin{array}{c}\text { Feed rate } \\
x_{3}(\mathrm{~mm} / \mathrm{rev})\end{array}$ \\
\hline 1 & 150 & 0.10 & 0.050 \\
2 & 175 & 0.15 & 0.075 \\
3 & 200 & 0.20 & 0.100 \\
4 & 250 & 0.30 & 0.150 \\
5 & 300 & 0.40 & 0.200 \\
\hline
\end{tabular}

\section{Full-Factorial Sampling}

A five-level full-factorial experimental matrix was used to explore various settings of the machining parameters. To frame the experiments within an optimization framework, the three machining parameters of cutting speed, depth of cut, and feed rate are referred to as the design variables $x_{1}$, $x_{2}$, and $x_{3}$, respectively. Additionally, the two performance measures of interest (surface quality and material removal rate) are formulated in a minimization setting, so that the objective is to minimize:

$f_{1}$ is the arithmetic average deviation of the assessed machined surface profile $(\mu \mathrm{m})$.

$f_{2}$ is machining time needed to remove a unit volume of the J-Steel material $\left(\mathrm{min} / \mathrm{cm}^{3}\right)$.

Listing of the five-level settings of the design variables is provided in Table 2. For three design variables, the number of samples corresponding to five levels is $5^{3}=125$. The corresponding objective values are plotted in Figure 2 and full listing of all 125 samples is provided in the appendix.

As the general consensus is that surface roughness in turning operations has high sensitivity to the feed rate [12], Figure 2 was constructed with separate markers for samples 
TABLE 3: Listing of undominated samples from full-factorial matrix.

\begin{tabular}{|c|c|c|c|c|c|c|}
\hline Pareto ID & $\begin{array}{c}\text { ID \# among } 125 \\
\text { samples }\end{array}$ & $x_{1}(\mathrm{~m} / \mathrm{min})$ & $x_{2}(\mathrm{~mm})$ & $x_{3}(\mathrm{~mm} / \mathrm{rev})$ & $f_{1}(\mu \mathrm{m})$ & $f_{2}\left(\mathrm{~min} / \mathrm{cm}^{3}\right)$ \\
\hline P1 & 56 & 200 & 0.15 & 0.050 & 0.264 & 0.66667 \\
\hline P2 & 36 & 175 & 0.20 & 0.050 & 0.296 & 0.57143 \\
\hline $\mathrm{P} 3^{*}$ & 66 & 200 & 0.30 & 0.050 & 0.309 & 0.33333 \\
\hline $\mathrm{P} 4^{*}$ & 47 & 175 & 0.40 & 0.075 & 0.381 & 0.19048 \\
\hline $\mathrm{P}^{*}$ & 121 & 300 & 0.40 & 0.050 & 0.504 & 0.16667 \\
\hline $\mathrm{P}^{*}$ & 98 & 250 & 0.40 & 0.100 & 0.518 & 0.10000 \\
\hline P7 & 123 & 300 & 0.40 & 0.100 & 0.801 & 0.08333 \\
\hline P8 & 99 & 250 & 0.40 & 0.150 & 1.222 & 0.06667 \\
\hline P9 & 124 & 300 & 0.40 & 0.150 & 1.963 & 0.05556 \\
\hline P10 & 125 & 300 & 0.40 & 0.200 & 2.861 & 0.04167 \\
\hline
\end{tabular}

"Sample is within the "knee-cap" zone of the Pareto frontier.

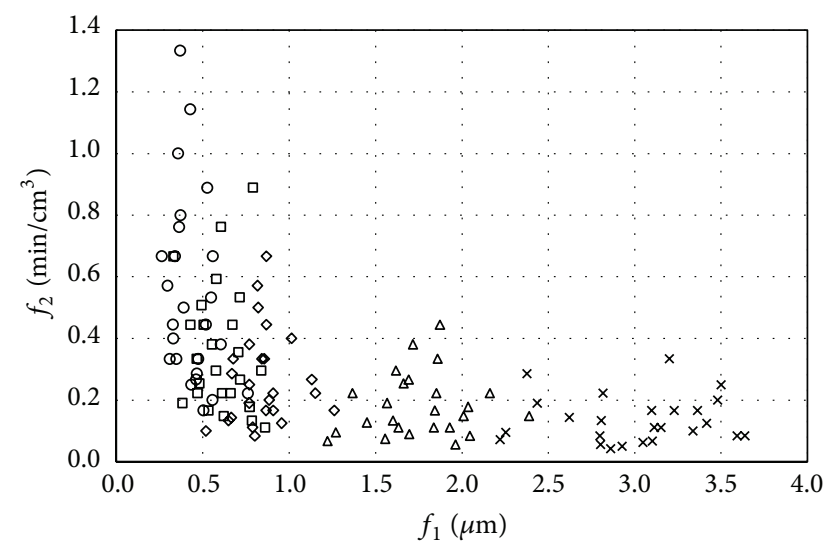

Samples at feed rate $(\mathrm{mm} / \mathrm{rev})$

- $0.050 \quad \Delta 0.150$

口 $0.075 \times 0.200$

$\diamond 0.100$

Figure 2: Objective values for five-level full-factorial samples.

that have different feed rate values. And while this consensus is observed to be mostly true in Figure 2 (samples with the same feed rate tend to cluster together), the following observations are also noted:

(i) For any given feed rate, there is a relatively large range of variation in both surface roughness $\left(f_{1}\right)$ and the machining time $\left(f_{2}\right)$. Thus, identification of the proper combination of cutting speed and depth of cut to match the selected feed rate is of significant importance.

(ii) There is quite a bit of overlap between clusters of same feed rate samples, especially at the submicron range for values of $\left(f_{1}\right)$. This implies that the proper (simultaneous) selection of cutting parameters is of higher significance than simply adjusting the parameter with high sensitivity.

Extracting the "undominated" trade-off frontier results in the Pareto plot shown in Figure 3. In Pareto analysis, a sample

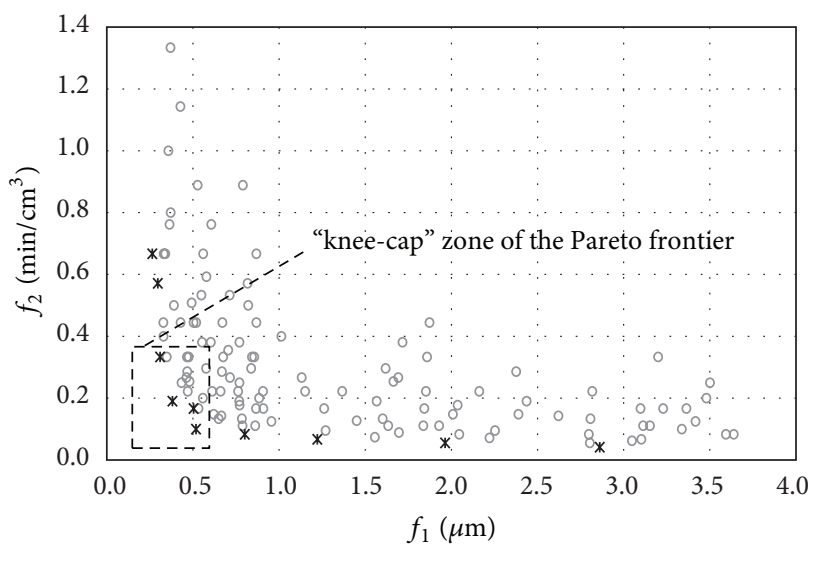

- Dominated samples

* Pareto front

Figure 3: Pareto plot for five-level full-factorial samples.

is considered "dominated" if another sample exists that has better value in one objective and no-worse values among all the objectives. In two-objective minimization problems, this dominance criterion translates to a sample being dominated if another sample exists towards its lower left in the Pareto plot. Listing of the undominated samples is provided in Table 3.

An important observation in Figure 3 is that the Pareto frontier appears to be "knee-shaped," which is one of the interesting types of multiobjective problems [13]. In these types of problems, the "knee-cap" of the Pareto frontier (highlighted in Figure 3) is where a process planner would likely focus their attention because this is where meaningful tradeoff decisions can be made. Excluding sample P5 (because it is near-dominated by $\mathrm{P} 6$ ), there are reasonable gains in machining time at the expense of moderate compromise in surface roughness by going from $\mathrm{P} 3$ to $\mathrm{P} 4$ to $\mathrm{P} 6$. On the other hand, outside of the knee-cap zone, there is very little gain in machining time at the expense of large compromise in surface roughness by going from P6 to P7-P10. Likewise, there is 
very little gain in surface roughness at large compromise in machining time by going from P3 to P1-P2.

\section{Multiobjective Efficient Global Optimization}

When full-factorial sampling with large number of levels is feasible in terms of cost and time to conduct the experiments, it is typically the approach of choice since it provides assurance that the entire space of tunable parameters has been explored. When the number of experiments needed becomes large (e.g., exponential growth of the number of samples becomes prohibitive when there are more than three tunable parameters or if the same process needs to be repeated for different tool/inserts), it is often desirable to seek approaches that focus on generating samples in the vicinity of the Pareto frontier instead of exploring the entire space of tunable parameters.

This section explores the "what-if" scenario of how many experimental samples would have been needed to generate a Pareto plot like Figure 3 by utilizing an alternative approach from the literature on metamodeling based design optimization. Metamodeling based design optimization, as described in the review articles in [14, 15], includes approaches that utilize (computationally inexpensive) numerical approximation models to what is otherwise a highly time/cost expensive process to model. The approximate models are then used to "guide" the sampling within the expensive process to what is predicted to be samples with more desirable performance. The approach adopted in this paper is an extension of Jones' "Efficient Global Optimization" (EGO) algorithm [5], which has been further developed and applied in many application studies [6-8]. Multiobjective versions of EGO were also developed, such as the work in $[9,10]$. A brief recap of the adopted algorithm is provided in the next subsection. For full details of the most recent version of the algorithm, readers are referred to [11].

4.1. The m-EGO Algorithm. All variations of the EGO algorithm use Kriging [16] metamodels. Like all metamodels, Kriging provides a prediction $\phi(\mathbf{x})$ that approximates time/costly to evaluate process $f(\mathbf{x})$, with $\mathbf{x}$ being a vector of design variables and $\phi$ and $f$ being the approximate and true process response, respectively. This prediction is done after "training" the metamodel to a number $N$ of known samples $\mathbf{x}^{1}, \mathbf{x}^{2}, \ldots, \mathbf{x}^{N}$ and their corresponding values of $f^{1}, f^{2}, \ldots, f^{N}$. However, what is rather unique in Kriging compared to other metamodels is that it also provides an expected variation $\sigma(\mathbf{x})$, which can also be interpreted as a measure of uncertainty in the predicted values $\phi(\mathbf{x})$. This allows an optimization algorithm to make predictions about the expected limits for the process output (regions with good expected improvement become candidates for exploring new samples) at various points within the search space, as well as identifying which regions of the search space have too low density of samples (and these locations also become candidates for sampling in order to further refine the metamodel). At a high level, the basic steps of an EGO algorithm are as follows:

(1) Train a Kriging model using known samples.

(2) Solve an "Infill Optimization Problem" using the Kriging model predictions $\phi(\mathbf{x}) \& \sigma(\mathbf{x})$ to generate new sample point(s).

(3) Evaluate the real process $f(\mathbf{x})$ for the new sample points.

(4) Add the new sample points to the set of known samples.

(5) Repeat step 1 unless a termination criterion is met.

(6) Output the best sample point(s).

Primary differences between different implementations of the EGO algorithm are usually in step 2, in particular,

(1) objective(s) for the infill optimization problem,

(2) optimization algorithm to solve the infill problem,

(3) whether new sample points are generated one at a time or in batches.

Other minor differences in implementation of the EGO algorithm can include the initial set of samples (which can be random or structured), as well as the termination criteria (which may be things like reaching a target process output, preset budget on number of samples, or number of iterations without new improved samples).

The m-EGO algorithm (as implemented in $[10,11])$ formulates the infill problem as an augmented multiobjective problem that seeks to minimize $\{\phi(\mathbf{x}),-\sigma(\mathbf{x})\}$ and solves it via the evolutionary optimization algorithm SPEA2 [17]. The output of SPEA2 is an "archive" of points distributed on the trade-off frontier of the Kriging model predictions, as well as regions of the search space with high uncertainty. A clustering technique is then applied to the archive of points in order to select a batch of new candidate sample points.

4.2. Implementation and Results. The m-EGO algorithm was tested to see if it would be successful in generating the Pareto trade-off frontier for the same ranges of tunable cutting parameters. Details of m-EGO implementation in this study are as follows:

(i) Structured initial set of samples, using four-level full factorial (64 initial samples). Listing of the design variables at different levels is provided in Table 4.

(ii) Termination condition which is upon reaching a preset budget of 80 experimental samples.

(iii) New samples generated in batches of 8 (thus the termination condition is met after two iterations of the algorithm, since $64+2 \times 8=80$ ).

Since this is a "what-if" study, the four levels in the fullfactor initial set of samples were selected to coincide with samples that were already experimented in the five-level fullfactorial study in Section 3 (relieving the need to conduct 
TABLE 4: Design variables values in four-level full-factorial matrix used as initial samples for m-EGO.

\begin{tabular}{lccc}
\hline Level & $\begin{array}{c}\text { Cutting speed } \\
x_{1}(\mathrm{~m} / \mathrm{min})\end{array}$ & $\begin{array}{c}\text { Depth of cut } \\
x_{2}(\mathrm{~mm})\end{array}$ & $\begin{array}{c}\text { Feed rate } \\
x_{3}(\mathrm{~mm} / \mathrm{rev})\end{array}$ \\
\hline 1 & 150 & 0.10 & 0.050 \\
2 & 200 & 0.20 & 0.100 \\
3 & 250 & 0.30 & 0.150 \\
4 & 300 & 0.40 & 0.200 \\
\hline
\end{tabular}

TABLE 5: Listing of undominated samples from m-EGO.

\begin{tabular}{lccccc}
\hline Pareto ID & $\begin{array}{c}x_{1} \\
(\mathrm{~m} / \mathrm{min})\end{array}$ & $x_{2}(\mathrm{~mm})$ & $\begin{array}{c}x_{3} \\
(\mathrm{~mm} / \mathrm{rev})\end{array}$ & $f_{1}(\mu \mathrm{m})$ & $\begin{array}{c}f_{2} \\
\left(\mathrm{~min} / \mathrm{cm}^{3}\right)\end{array}$ \\
\hline E1 $^{*}$ & 200 & 0.30 & 0.050 & 0.309 & 0.33333 \\
E2 $^{*}$ & 223 & 0.31 & 0.050 & 0.374 & 0.28695 \\
E3 $^{*}$ & 200 & 0.40 & 0.050 & 0.434 & 0.25000 \\
E4 $^{*}$ & 259 & 0.40 & 0.050 & 0.443 & 0.19279 \\
E5 $^{*}$ & 300 & 0.40 & 0.050 & 0.504 & 0.16667 \\
E6 & 250 & 0.40 & 0.100 & 0.518 & 0.10000 \\
E7 & 300 & 0.40 & 0.100 & 0.801 & 0.08333 \\
E8 & 250 & 0.40 & 0.150 & 1.222 & 0.06667 \\
E9 & 300 & 0.40 & 0.150 & 1.963 & 0.05556 \\
E10 & 300 & 0.40 & 0.200 & 2.861 & 0.04167 \\
\hline
\end{tabular}

*Sample is within the "knee-cap" zone of the Pareto frontier.

64 new experiments). New experiments were conducted to evaluate the performance of the 16 new samples generated by two iterations of the algorithm and are shown in Figure 4. It is notable in Figure 4 how the newly generated samples are mostly biased towards the Pareto frontier (implying that the $\mathrm{m}$-EGO algorithm does in fact work as intended), with some of the new samples leading to discovery of new undominated samples, two of which within the knee-cap zone of the Pareto frontier. Listing of the undominated samples obtained via the approach is provided in Table 5.

\section{Discussion}

J-Steels have relatively high hardness that correlates well with good surface quality in machining processes. This is mainly due to possessing low plastic flowability which results in better surface finish. This is attributed to the brittle nature of the interaction between the cutting tool and the workpiece surface, for the hard materials, that leads to material separation rather than plastic flow that would result in surface irregularities. Surface roughness was found to increase with increasing feed rate and depth of cut which both result in bigger cut areas that are consequently associated with higher cutting forces and higher friction which lead to poor surface finish. It was noticed from the surface roughness profile that high feed rates were associated with lager roughness

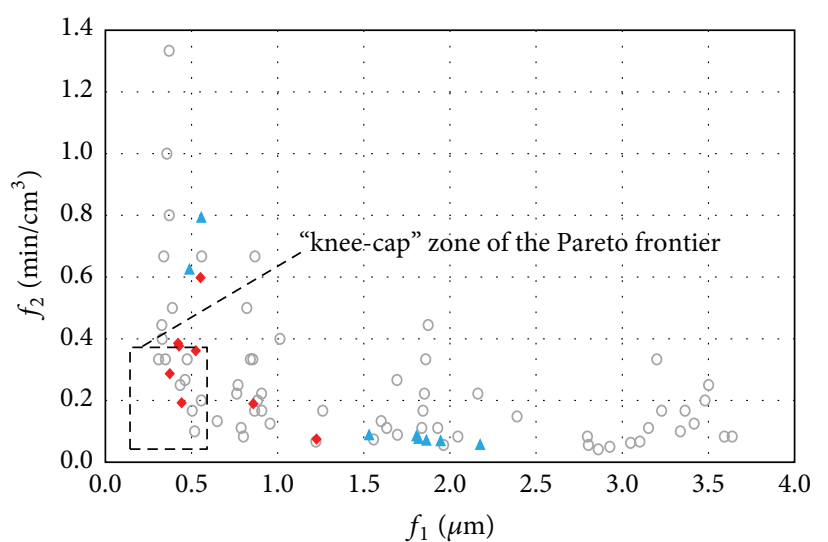

- Initial 64 samples
- m-EGO iteration 1
$\Delta$ m-EGO iteration 2

FIGURE 4: Objective values for samples obtained via m-EGO approach.

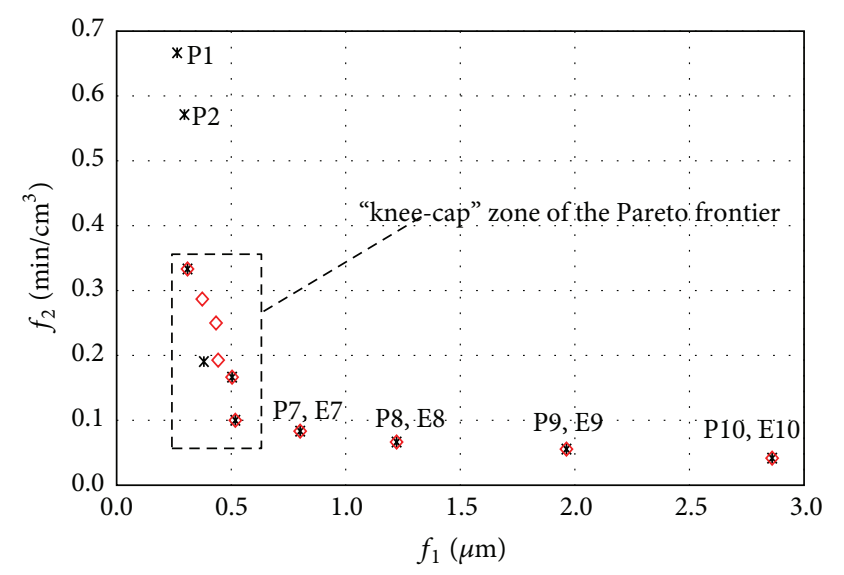

* Five-level full factorial

$\diamond \mathrm{m}-\mathrm{EGO}$

FIGURE 5: Comparison of obtained Pareto frontiers.

markings horizontal spacing. Also, with higher depth of cuts, the vertical spacing between peaks and troughs of the surface irregularities was larger. Thus, higher feed rates and depth of cuts generally lead to higher surface roughness. However, higher feed rates and depth of cut are favorable in terms of increasing material removal rate. Thus, there is good motivation for finding the right combination of settings of the cutting parameters in order to obtain a good compromise between surface quality and machining time. Search for this good compromise may be done via "dense sampling" (such as the five-level full-factorial design of experiments) or through optimization techniques (such as m-EGO).

A comparison of obtained Pareto frontiers via the two approaches (five-level full factorial and m-EGO) is shown in Figure 5. Noted observations include the following:

(i) Undominated samples to the right-side of the kneecap zone of the Pareto frontier (five-level full-factorial 
samples P7-P10 in Table 3) were exactly identified by the m-EGO approach (samples E7-E10 in Table 5).

(ii) Undominated samples to the top of the knee-cap zone of the Pareto frontier (five-level full-factorial samples P1, P2 in Table 3) were not identified by the m-EGO approach.

(iii) Sample P4 (from five-level full factorial) is dominant with respect to samples E3 and E4 (from m-EGO). However, sample E4 is fairly close in performance to $\mathrm{P} 4$.

(iv) m-EGO approach achieved a higher density of Pareto points (more alternative choices) within the knee-cap zone of the Pareto frontier, which is the primary area of interest for making trade-off decisions.

Overall, neither approach is decisively better in terms of quality of the generated Pareto frontiers. However, the m-EGO approach only required 80 sample experiments, while the five-level full factorial required 125 experiments. Thus, m-EGO achieved a comparable quality Pareto frontier for tuning the cutting parameters with 36\% fewer experiments. It is also notable that this study presents the first demonstrated success of m-EGO with real machining experiments, as opposed to previous m-EGO studies $[10,11]$ that used numerical model/simulations of the machining processes.

\section{Conclusion}

This paper presented a multiobjective optimization study for tuning the cutting parameters in turning operation of J-Steel material. Exploration of the search space was conducted via five-level full factorial, as well as a metamodeling based optimization approach (m-EGO). Both approaches had qualitatively similar results that revealed the Pareto trade-off frontier as having a knee-shape, where the kneecap zone becomes the primary area of interest for reasonable trade-off decisions. The knee-cap zone included trade-off settings of the machining parameters that range from $\mathrm{Ra}$ surface roughness of $0.32 \mu \mathrm{m}$ to $0.52 \mu \mathrm{m}$ at machining time of $0.25 \mathrm{~min} / \mathrm{cm}^{3}$ to $0.1 \mathrm{~min} / \mathrm{cm}^{3}$, respectively. Testing of the $\mathrm{m}-$ EGO approach was demonstrated as a success in generating the Pareto frontier with $36 \%$ fewer sample experiments, which is sizable saving in time and resources. The m-EGO approach is thus recommended for similar future studies that would seek to tune cutting parameters for different tools and/or materials.

\section{Appendix}

See Table 6.

\section{Competing Interests}

The authors declare that they have no competing interests.
TABLE 6: Listing of five-level full-factorial samples.

\begin{tabular}{|c|c|c|c|c|c|}
\hline ID $\# x_{1}$ & $(\mathrm{~m} / \mathrm{min})$ & $x_{2}(\mathrm{~mm})$ & $x_{3}(\mathrm{~mm} / \mathrm{rev})$ & $f_{1}(\mu \mathrm{m})$ & $f_{2}\left(\mathrm{~min} / \mathrm{cm}^{3}\right)$ \\
\hline 1 & 150 & 0.10 & 0.050 & 0.370 & 1.3333 \\
\hline 2 & 150 & 0.10 & 0.075 & 0.790 & 0.8889 \\
\hline 3 & 150 & 0.10 & 0.100 & 0.868 & 0.6667 \\
\hline 4 & 150 & 0.10 & 0.150 & 1.873 & 0.4444 \\
\hline 5 & 150 & 0.10 & 0.200 & 3.200 & 0.3333 \\
\hline 6 & 150 & 0.15 & 0.050 & 0.527 & 0.8889 \\
\hline 7 & 150 & 0.15 & 0.075 & 0.578 & 0.5926 \\
\hline 8 & 150 & 0.15 & 0.100 & 0.868 & 0.4444 \\
\hline 9 & 150 & 0.15 & 0.150 & 1.618 & 0.2963 \\
\hline 10 & 150 & 0.15 & 0.200 & 2.817 & 0.2222 \\
\hline 11 & 150 & 0.20 & 0.050 & 0.339 & 0.6667 \\
\hline 12 & 150 & 0.20 & 0.075 & 0.504 & 0.4444 \\
\hline 13 & 150 & 0.20 & 0.100 & 0.856 & 0.3333 \\
\hline 14 & 150 & 0.20 & 0.150 & 2.162 & 0.2222 \\
\hline 15 & 150 & 0.20 & 0.200 & 3.229 & 0.1667 \\
\hline 16 & 150 & 0.30 & 0.050 & 0.328 & 0.4444 \\
\hline 17 & 150 & 0.30 & 0.075 & 0.576 & 0.2963 \\
\hline 18 & 150 & 0.30 & 0.100 & 0.907 & 0.2222 \\
\hline 19 & 150 & 0.30 & 0.150 & 2.389 & 0.1481 \\
\hline 20 & 150 & 0.30 & 0.200 & 3.152 & 0.1111 \\
\hline 21 & 150 & 0.40 & 0.050 & 0.348 & 0.3333 \\
\hline 22 & 150 & 0.40 & 0.075 & 0.660 & 0.2222 \\
\hline 23 & 150 & 0.40 & 0.100 & 1.261 & 0.1667 \\
\hline 24 & 150 & 0.40 & 0.150 & 1.929 & 0.1111 \\
\hline 25 & 150 & 0.40 & 0.200 & 2.798 & 0.0833 \\
\hline 26 & 175 & 0.10 & 0.050 & 0.427 & 1.1429 \\
\hline 27 & 175 & 0.10 & 0.075 & 0.606 & 0.7619 \\
\hline 28 & 175 & 0.10 & 0.100 & 0.817 & 0.5714 \\
\hline 29 & 175 & 0.10 & 0.150 & 1.716 & 0.3810 \\
\hline 30 & 175 & 0.10 & 0.200 & 2.376 & 0.2857 \\
\hline 31 & 175 & 0.15 & 0.050 & 0.364 & 0.7619 \\
\hline 32 & 175 & 0.15 & 0.075 & 0.492 & 0.5079 \\
\hline 33 & 175 & 0.15 & 0.100 & 0.770 & 0.3810 \\
\hline 34 & 175 & 0.15 & 0.150 & 1.663 & 0.2540 \\
\hline 35 & 175 & 0.15 & 0.200 & 2.436 & 0.1905 \\
\hline 36 & 175 & 0.20 & 0.050 & 0.296 & 0.5714 \\
\hline 37 & 175 & 0.20 & 0.075 & 0.553 & 0.3810 \\
\hline 38 & 175 & 0.20 & 0.100 & 0.668 & 0.2857 \\
\hline 39 & 175 & 0.20 & 0.150 & 1.566 & 0.1905 \\
\hline 40 & 175 & 0.20 & 0.200 & 2.621 & 0.1429 \\
\hline 41 & 175 & 0.30 & 0.050 & 0.604 & 0.3810 \\
\hline 42 & 175 & 0.30 & 0.075 & 0.480 & 0.2540 \\
\hline 43 & 175 & 0.30 & 0.100 & 0.769 & 0.1905 \\
\hline 44 & 175 & 0.30 & 0.150 & 1.450 & 0.1270 \\
\hline 45 & 175 & 0.30 & 0.200 & 2.253 & 0.0952 \\
\hline 46 & 175 & 0.40 & 0.050 & 0.467 & 0.2857 \\
\hline 47 & 175 & 0.40 & 0.075 & 0.381 & 0.1905 \\
\hline 48 & 175 & 0.40 & 0.100 & 0.665 & 0.1429 \\
\hline 49 & 175 & 0.40 & 0.150 & 1.270 & 0.0952 \\
\hline
\end{tabular}


TABle 6: Continued.

\begin{tabular}{|c|c|c|c|c|c|}
\hline ID \# $x$ & $(\mathrm{~m} / \mathrm{min})$ & $x_{2}(\mathrm{~mm})$ & $x_{3}(\mathrm{~mm} / \mathrm{rev})$ & $f_{1}(\mu \mathrm{m})$ & $f_{2}\left(\mathrm{~min} / \mathrm{cm}^{3}\right)$ \\
\hline 50 & 175 & 0.40 & 0.200 & 2.221 & 0.0714 \\
\hline 51 & 200 & 0.10 & 0.050 & 0.357 & 1.0000 \\
\hline 52 & 200 & 0.10 & 0.075 & 0.330 & 0.6667 \\
\hline 53 & 200 & 0.10 & 0.100 & 0.821 & 0.5000 \\
\hline 54 & 200 & 0.10 & 0.150 & 1.859 & 0.3333 \\
\hline 55 & 200 & 0.10 & 0.200 & 3.501 & 0.2500 \\
\hline 56 & 200 & 0.15 & 0.050 & 0.264 & 0.6667 \\
\hline 57 & 200 & 0.15 & 0.075 & 0.428 & 0.4444 \\
\hline 58 & 200 & 0.15 & 0.100 & 0.675 & 0.3333 \\
\hline 59 & 200 & 0.15 & 0.150 & 1.366 & 0.2222 \\
\hline 60 & 200 & 0.15 & 0.200 & 3.098 & 0.1667 \\
\hline 61 & 200 & 0.20 & 0.050 & 0.389 & 0.5000 \\
\hline 62 & 200 & 0.20 & 0.075 & 0.465 & 0.3333 \\
\hline 63 & 200 & 0.20 & 0.100 & 0.770 & 0.2500 \\
\hline 64 & 200 & 0.20 & 0.150 & 1.843 & 0.1667 \\
\hline 65 & 200 & 0.20 & 0.200 & 3.417 & 0.1250 \\
\hline 66 & 200 & 0.30 & 0.050 & 0.309 & 0.3333 \\
\hline 67 & 200 & 0.30 & 0.075 & 0.470 & 0.2222 \\
\hline 68 & 200 & 0.30 & 0.100 & 0.908 & 0.1667 \\
\hline 69 & 200 & 0.30 & 0.150 & 1.837 & 0.1111 \\
\hline 70 & 200 & 0.30 & 0.200 & 3.593 & 0.0833 \\
\hline 71 & 200 & 0.40 & 0.050 & 0.434 & 0.2500 \\
\hline 72 & 200 & 0.40 & 0.075 & 0.532 & 0.1667 \\
\hline 73 & 200 & 0.40 & 0.100 & 0.956 & 0.1250 \\
\hline 74 & 200 & 0.40 & 0.150 & 2.046 & 0.0833 \\
\hline 75 & 200 & 0.40 & 0.200 & 3.048 & 0.0625 \\
\hline 76 & 250 & 0.10 & 0.050 & 0.371 & 0.8000 \\
\hline 77 & 250 & 0.10 & 0.075 & 0.713 & 0.5333 \\
\hline 78 & 250 & 0.10 & 0.100 & 1.013 & 0.4000 \\
\hline 79 & 250 & 0.10 & 0.150 & 1.692 & 0.2667 \\
\hline 80 & 250 & 0.10 & 0.200 & 3.480 & 0.2000 \\
\hline 81 & 250 & 0.15 & 0.050 & 0.549 & 0.5333 \\
\hline 82 & 250 & 0.15 & 0.075 & 0.705 & 0.3556 \\
\hline 83 & 250 & 0.15 & 0.100 & 1.131 & 0.2667 \\
\hline 84 & 250 & 0.15 & 0.150 & 2.036 & 0.1778 \\
\hline 85 & 250 & 0.15 & 0.200 & 2.807 & 0.1333 \\
\hline 86 & 250 & 0.20 & 0.050 & 0.329 & 0.4000 \\
\hline 87 & 250 & 0.20 & 0.075 & 0.715 & 0.2667 \\
\hline 88 & 250 & 0.20 & 0.100 & 0.884 & 0.2000 \\
\hline 89 & 250 & 0.20 & 0.150 & 1.601 & 0.1333 \\
\hline 90 & 250 & 0.20 & 0.200 & 3.337 & 0.1000 \\
\hline 91 & 250 & 0.30 & 0.050 & 0.462 & 0.2667 \\
\hline 92 & 250 & 0.30 & 0.075 & 0.771 & 0.1778 \\
\hline 93 & 250 & 0.30 & 0.100 & 0.649 & 0.1333 \\
\hline 94 & 250 & 0.30 & 0.150 & 1.695 & 0.0889 \\
\hline 95 & 250 & 0.30 & 0.200 & 3.102 & 0.0667 \\
\hline 96 & 250 & 0.40 & 0.050 & 0.557 & 0.2000 \\
\hline 97 & 250 & 0.40 & 0.075 & 0.784 & 0.1333 \\
\hline 98 & 250 & 0.40 & 0.100 & 0.518 & 0.1000 \\
\hline 99 & 250 & 0.40 & 0.150 & 1.222 & 0.0667 \\
\hline 100 & 250 & 0.40 & 0.200 & 2.928 & 0.0500 \\
\hline
\end{tabular}

TABLE 6: Continued.

\begin{tabular}{|c|c|c|c|c|c|}
\hline ID $\# x$ & $(\mathrm{~m} / \mathrm{min})$ & $x_{2}(\mathrm{~mm})$ & $x_{3}(\mathrm{~mm} / \mathrm{rev})$ & $f_{1}(\mu \mathrm{m})$ & $f_{2}\left(\mathrm{~min} / \mathrm{cm}^{3}\right)$ \\
\hline 101 & 300 & 0.10 & 0.050 & 0.559 & 0.6667 \\
\hline 102 & 300 & 0.10 & 0.075 & 0.671 & 0.4444 \\
\hline 103 & 300 & 0.10 & 0.100 & 0.842 & 0.3333 \\
\hline 104 & 300 & 0.10 & 0.150 & 1.852 & 0.2222 \\
\hline 105 & 300 & 0.10 & 0.200 & 3.364 & 0.1667 \\
\hline 106 & 300 & 0.15 & 0.050 & 0.519 & 0.4444 \\
\hline 107 & 300 & 0.15 & 0.075 & 0.838 & 0.2963 \\
\hline 108 & 300 & 0.15 & 0.100 & 1.151 & 0.2222 \\
\hline 109 & 300 & 0.15 & 0.150 & 2.009 & 0.1481 \\
\hline 110 & 300 & 0.15 & 0.200 & 3.113 & 0.1111 \\
\hline 111 & 300 & 0.20 & 0.050 & 0.475 & 0.3333 \\
\hline 112 & 300 & 0.20 & 0.075 & 0.611 & 0.2222 \\
\hline 113 & 300 & 0.20 & 0.100 & 0.866 & 0.1667 \\
\hline 114 & 300 & 0.20 & 0.150 & 1.633 & 0.1111 \\
\hline 115 & 300 & 0.20 & 0.200 & 3.638 & 0.0833 \\
\hline 116 & 300 & 0.30 & 0.050 & 0.762 & 0.2222 \\
\hline 117 & 300 & 0.30 & 0.075 & 0.621 & 0.1481 \\
\hline 118 & 300 & 0.30 & 0.100 & 0.788 & 0.1111 \\
\hline 119 & 300 & 0.30 & 0.150 & 1.556 & 0.0741 \\
\hline 120 & 300 & 0.30 & 0.200 & 2.803 & 0.0556 \\
\hline 121 & 300 & 0.40 & 0.050 & 0.504 & 0.1667 \\
\hline 122 & 300 & 0.40 & 0.075 & 0.861 & 0.1111 \\
\hline 123 & 300 & 0.40 & 0.100 & 0.801 & 0.0833 \\
\hline 124 & 300 & 0.40 & 0.150 & 1.963 & 0.0556 \\
\hline 125 & 300 & 0.40 & 0.200 & 2.861 & 0.0417 \\
\hline
\end{tabular}

\section{Acknowledgments}

This Project was supported by King Saud University, Deanship of Scientific Research, College of Engineering Research Center.

\section{References}

[1] V. N. Gaitonde, S. R. Karnik, L. Figueira, and J. Paulo-Davim, "Machinability investigations in hard turning of AISI D2 cold work tool steel with conventional and wiper ceramic inserts," International Journal of Refractory Metals \& Hard Materials, vol. 27, no. 4, pp. 754-763, 2009.

[2] A. K. Sahoo, A. N. Baral, A. K. Rout, and B. C. Routra, "Multiobjective optimization and predictive modeling of surface roughness and material removal rate in turning using grey relational and regression analysis," Procedia Engineering, vol. 38, pp. 1606-1627, 2012.

[3] A. Srithar, K. Palanikumar, and B. Durgaprasad, "Experimental investigation and surface roughness analysis on hard turning of AISI D2 steel using coated carbide insert," Procedia Engineering, vol. 97, pp. 72-77, 2014.

[4] R. Suresh, S. Basavarajappa, V. N. Gaitonde, G. L. Samuel, and J. P. Davim, "State-of-the-art research in machinability of hardened steels," Proceedings of the Institution of Mechanical Engineers, Part B: Journal of Engineering Manufacture, vol. 227, no. 2, pp. 191-209, 2013. 
[5] D. R. Jones, M. Schonlau, and W. J. Welch, "Efficient global optimization of expensive black-box functions," Journal of Global Optimization, vol. 13, no. 4, pp. 455-492, 1998.

[6] M. J. Sasena, P. Papalambros, and P. Goovaerts, "Exploration of metamodeling sampling criteria for constrained global optimization," Engineering Optimization, vol. 34, no. 3, pp. 263-278, 2002.

[7] M. J. Sasena, M. Parkinson, M. P. Reed, P. Y. Papalambros, and P. Goovaerts, "Improving an ergonomics testing procedure via approximation based adaptive experimental design," Journal of Mechanical Design, vol. 127, no. 5, pp. 1006-1013, 2005.

[8] Y. Ren and P. Y. Papalambros, "Design preference elicitation using efficient global optimization," in Proceedings of the ASME 2011 International Design Engineering Technical Conferences and Computers and Information in Engineering Conference (IDETC/CIE '11), pp. 591-600, Washington, Wash, USA, August 2011.

[9] J. Knowles, "ParEGO: a hybrid algorithm with on-line landscape approximation for expensive multiobjective optimization problems," IEEE Transactions on Evolutionary Computation, vol. 10, no. 1, pp. 50-66, 2006.

[10] M. Aly, K. Hamza, M. Tauhiduzzaman et al., "Multi-objective selection of cutting conditions in advanced machining processes via an efficient global optimization approach," in Proceedings of the International Design Engineering Technical Conferences and Computers and Information in Engineering Conference (ASME '14), Paper No. DETC2014-34624, 8 pages, Buffalo, NY, USA, August 2014.

[11] A. Sadek, M. Aly, K. Hamza, M. Meshreki, A. O. Nassef, and H. Attia, "Optimization of cutting conditions in vibration assisted drilling of composites via a multi-objective EGO implementation," in Proceedings of the ASME International Design Engineering Technical Conferences (ASME IDETC '15), Paper no. DETC2015-47532, pp. 1-9, Boston, Mass, USA, 2015.

[12] S. Coromant, “Turning Tools," 2012, http://www.sandvik.coromant.com/sitecollectiondocuments/downloads/global/catalogues/en-gb/turning/turn_g.pdf.

[13] K. Deb, Multi-Objective Optimization Using Evolutionary Algorithms, John Wiley \& Sons, Hoboken, NJ, USA, 2003.

[14] T. W. Simpson, A. J. Booker, D. Ghosh, A. A. Giunta, P. N. Koch, and R.-J. Yang, "Approximation methods in multidisciplinary analysis and optimization: a panel discussion," Structural and Multidisciplinary Optimization, vol. 27, no. 5, pp. 302-313, 2004.

[15] F. Viana, C. Gogu, and R. Haftka, "Making the most out of surrogate models: tricks of the trade," in Proceedings of the ASME International Design Engineering Technical Conferences (IDETC '10), DETC2010-28064, Montreal, Canada, 2010.

[16] P. Goovaerts, Geostatistics for Natural Evaluation, Oxford University Press, New York, NY, USA, 1997.

[17] E. Zitzler, M. Laumanns, and L. Thiele, "SPEA2: improving the strength pareto evolutionary algorithm," TIK-Report 103, Swiss Federal Institute of Technology, Zurich, Switzerland, 2001. 

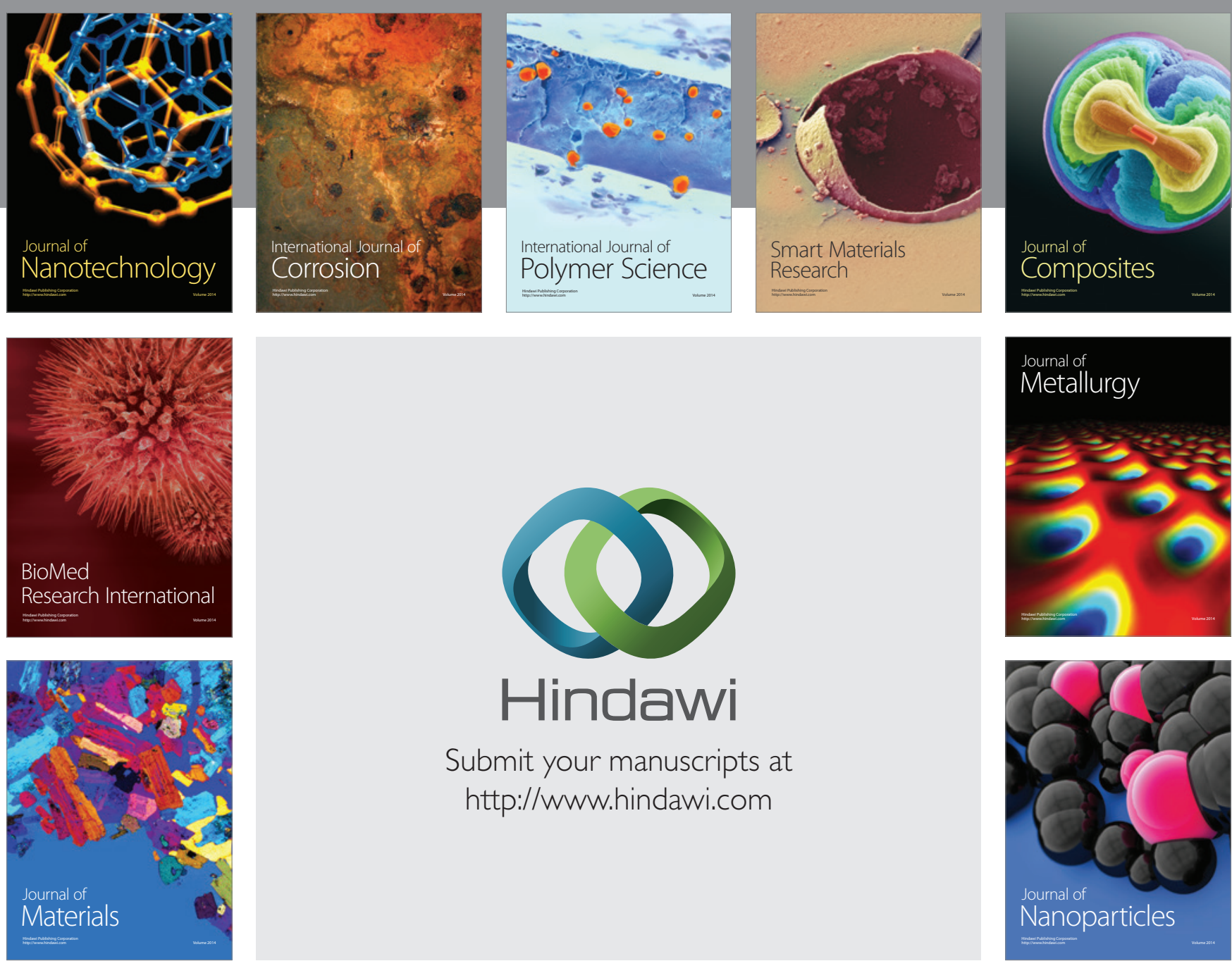

\section{Hindawi}

Submit your manuscripts at

http://www.hindawi.com

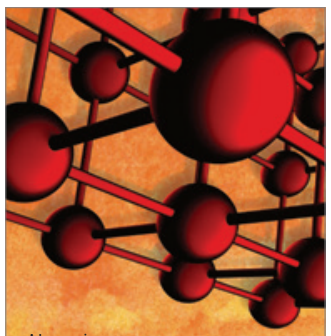

Materials Science and Engineering
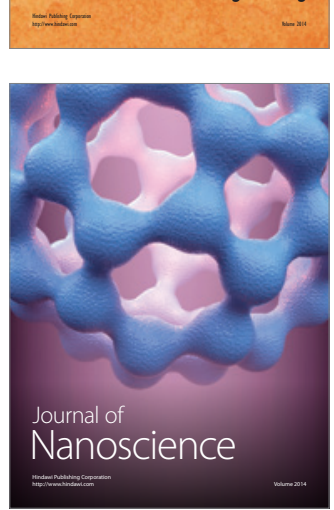
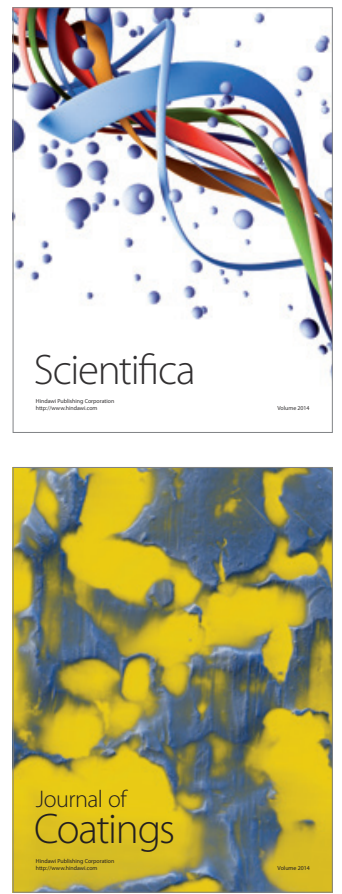
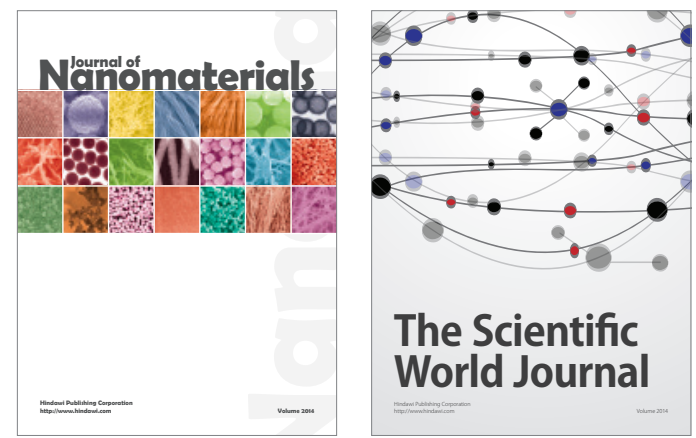

The Scientific World Journal
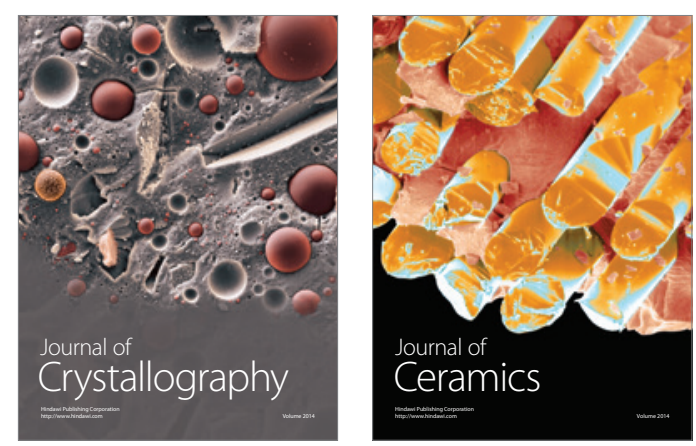
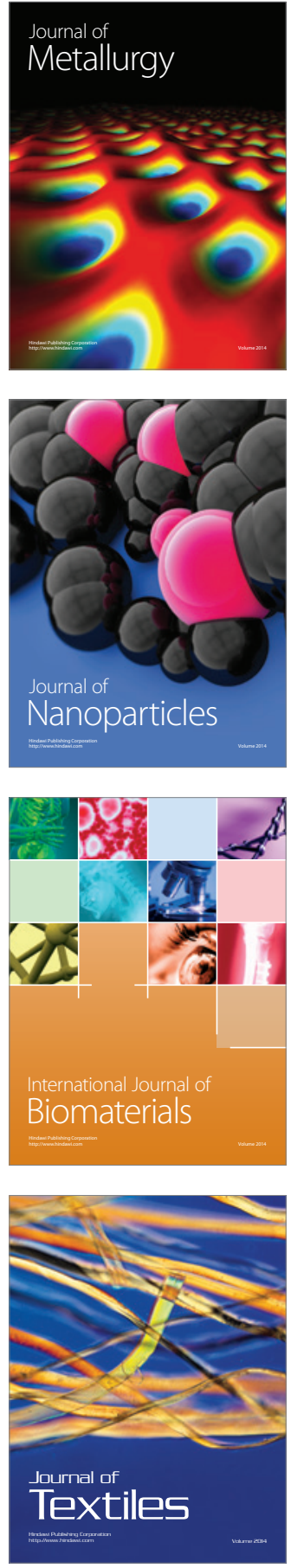